EGU21-9441

https://doi.org/10.5194/egusphere-egu21-9441

EGU General Assembly 2021

(c) Author(s) 2021. This work is distributed under

the Creative Commons Attribution 4.0 License.

\title{
Global Ionospheric Scintillation Model: current status and further development strategies
}

Dmytro Vasylyev ${ }^{1}$, Yannick Beniguel ${ }^{2}$, Volker Wilken ${ }^{1}$, Martin Kriegel ${ }^{1}$, and Jens Berdermann ${ }^{1}$ ${ }^{1}$ DLR Institute for Solar-Terrestrial Physics, Space Weather Impact, Germany

${ }^{2}$ Informatique Electromagnetisme Electronique Analyse Numerique, France

When a electromagnetic wave propagates through a random inhomogeneous medium, scattering by the refractive index inhomogeneities can lead to a wide variety of phenomena that have been the subject of extensive study and modelling. The Global Ionospheric Scintillation Model (GISM) is primarily intended to model the phenomena relevant for the GNSS applications and provides the amplitude and phase scintillation indices. Due to the three dimensional nature of the GISM model it is capable to describe a variety of communication geometries such as satellite-ground station or satellite-satellite communication link. Moreover, it can calculate the scintillation maps at specific altitude allowing to obtain the 3D picture of scintillation.

Recently the GISM model has been handed over to the newly established DLR Institute of SolarTerrestrial Physics. Since then the model underwent several modernization steps. For example, the programming paradigm has been changed to the object-oriented one in order to bring more flexibility into the code. In the present contribution we present the first results of our works and discuss strategies for further development, extension, and validation of the GISM. 